\title{
Functional NIRS study of blood brain barrier disruption when induced by focused ultrasound and intra-arterial mannitol infusion
}

Myllylä, Teemu, Kaakinen, Mika, Vihriälä, Erkki, Jukkola, Jari, Zhao, Zuomin, et al.

Teemu Myllylä, Mika Kaakinen, Erkki Vihriälä, Jari Jukkola, Zuomin Zhao, Hany Ferdinando, Vesa Korhonen, Vesa Kiviniemi, Lauri Eklund, "Functional NIRS study of blood brain barrier disruption when induced by focused ultrasound and intra-arterial mannitol infusion," Proc. SPIE 11363, Tissue Optics and Photonics, 113630T (5 May 2020); doi: 10.1117/12.2555843

SPIE. Event: SPIE Photonics Europe, 2020, Online Only, France 


\title{
Functional NIRS study of blood brain barrier disruption when induced by focused ultrasound and intra-arterial mannitol infusion
}

\author{
Teemu Myllylä*a,b, Mika Kaakinen ${ }^{\mathrm{c}}$, Erkki Vihriäläb, Jari Jukkolac ${ }^{\mathrm{c}}$, Zuomin Zhao ${ }^{\mathrm{b}}$, \\ Hany Ferdinando ${ }^{\text {a }}$, Vesa Korhonen ${ }^{\text {a,d }}$, Vesa Kiviniemi ${ }^{\text {a,d }}$, Lauri Eklund ${ }^{\mathrm{c}}$ \\ ${ }^{a}$ University of Oulu, Research Unit of Medical Imaging, Physics and Technology, Oulu, Finland; \\ ${ }^{b}$ University of Oulu, Optoelectronics and Measurement Techniques Unit, Oulu, Finland; \\ 'Biocenter Oulu, Faculty of Biochemistry and Molecular Medicine, Oulu, Finland; \\ ${ }^{\mathrm{d} O u l u}$ University Hospital, Department of Diagnostic Radiology, Medical Research Center (MRC), \\ Oulu, Finland
}

\begin{abstract}
Enhancing brain fluid movement across blood brain barrier (BBB) has been recognized as a potential treatment of neurodegenerative diseases. Moreover, BBB opening is of high interest also in brain drug delivery in the treatment of brain tumors/cancers. However, efficient therapies which are based on BBB opening are still limited because of insufficient understanding of mechanisms and safety issues. Currently, there are few promising methodologically diverse BBB opening approaches.

In this paper, we use functional near-infrared spectroscopy (fNIRS) for the first time for monitoring cerebral hemoglobin and water concentration changes during BBB opening in mouse brain by using two different techniques: intra-arterial mannitol infusion (IAM) and focused ultrasound (FUS). Both of these BBB opening techniques are already in clinical use but their hemo- and hydrodynamic implications have not been investigated from comparative aspect. Two fibre detectors were attached on both sides of the mouse brain and the source fibre was attached on middle of forehead. Further, by using a combination of three wavelengths $690 \mathrm{~nm}, 830 \mathrm{~nm}$ and $980 \mathrm{~nm}$, that have sufficient light penetration in the mouse brain, we can show average dynamics of hemoglobin and water in the whole brain, synchronized with BBB opening. To validate the level of BBB opening we used Evans blue dye and show its accumulation in the brain parenchyma tissue with the corresponding fNIRS responses.
\end{abstract}

Keywords: BBB disruption, brain, cerebral hemodynamics, cerebrospinal fluid, glymphatic, mannitol, water.

\section{INTRODUCTION}

The blood-brain barrier (BBB) regulates the central nervous system (CNS) homeostasis, and allows proper neuronal function, as well as prevents toxins, pathogens and inflammation to affect the CNS [1]. However, for treatment of brain disease such as brain tumors, BBB opening is needed, to enable chemotherapy drug to penetrate into the CNS. In addition, increasing brain fluid movement and glymphatic activity by modulating the BBB has been recognized as a potential treatment method for neurodegenerative diseases [2]. For instance, findings on Alzheimer Disease mouse models indicate that increasing of BBB permeability with focused ultrasound (FUS) can facilitate the removal of the protein accumulations and reverse memory loss [3]. Consequently, development of efficient and safe BBB opening techniques and monitoring, understanding BBB mechanism and the brain drainage system are of high interest [4-8].

BBB opening is already clinically exploited in brain drug delivery by using FUS [9] and mannitol infusion [10, 11]. In FUS based BBB opening, microbubbles are excited by ultra sound energy and interactions of the bubbles with the endothelial wall primarily creates BBB opening [9]. Generally seen, intra-arterial mannitol (IAM) based BBB opening is primarily based on osmotic shrinkage of endothelial cells which consequently opens tight junctions between the cells [6, 12].

*teemu.myllyla@oulu.fi

Tissue Optics and Photonics, edited by Valery V. Tuchin, Walter C. P. M. Blondel,

Zeev Zalevsky, Proc. of SPIE Vol. 11363, 113630T · (c) 2020 SPIE

CCC code: $0277-786 \mathrm{X} / 20 / \$ 21 \cdot$ doi: $10.1117 / 12.2555843$ 
There is a large number of studies on FUS therapies. Biological effects of FUS can be invisible, visible or destructive, depending on the rate of acoustic energy deposition. In particular, transcranial FUS is non-invasive, making it suitable for wide range of therapies, where existing treatment options are lacking, limited or not suitable for all patients. Munoz et al. reviewed in 2018 recent developments in understanding of how FUS stimulates the brain, and concludes that effects mediated by BBB may stay open longer than FUS treatment, and because of this, future challenges include refining control of the duration of neural and behavioral effects [13]. There are also many preclinical and clinical studies showing that transiently disrupting the BBB with IAM during chemotherapy holds much promise as a therapeutic method. However, researchers still have limited understanding of the BBB opening mechanisms of both FUS and IAM and, in particular, how BBB disruption affects fluid exchange in the brain.

Only a limited number of studies are focusing on the hemodynamic effects of BBB opening. Yoon et al. used spectral range of $700 \mathrm{~nm}$ to $900 \mathrm{~nm}$, to quantify hemodynamic changes in brain cortex when stimulating mouse brain by FUS. Optical fibers were positioned on the mouse scalp at source-detector distance of $4 \mathrm{~mm}$. High frequency ( $8 \mathrm{MHz}$ ) FUS induced a pronounced increase in $\mathrm{HbO}$ and decrease in $\mathrm{HbR}$, showing a pattern similar to the general hemodynamic response to neural activity [14]. Moreover, some interesting studies using fMRI Blood oxygenation level dependent (BOLD) during FUS BBBD show that BOLD is increasing when FUS is applied, for example by Chu et al. [15]. Milej et al. [16] quantified BBB permeability using dynamic contrast-enhanced NIRS utilising indocyanine green (ICG, $67 \mathrm{kDa}$ ) and $800 \mathrm{CW}$ carboxylate (IRDye, $1166 \mathrm{kDa}$ ), and generated permeability surface-area maps. This study demonstrates that BBB breakdown (accidentally) may contribute to secondary brain injury and lead to vascular edema accompanied with infiltration of white blood cells. Todd et al. show that neurovascular response is attenuated by FUS BBB opening, particularly BOLD and cerebral blood flow (CBF) changes are suppressed in response to forepaw pinching stimulation [17].

Ergin et al. documented several physiological responses due to IAM and ICG [18]. Mannitol injection brought significant changes in heart rate, blood pressure, and $\mathrm{CBF}$, but the hemodynamic parameters return to normal after the injection. Myllylä et al. showed the cardiovascular effect of IAM in both human and mouse with comparable results: IAM causes strong immediate cardiovascular effects [19]. Recently, we presented a combined method using directcurrent electroencephalography (DC-EEG) and fNIRS for monitoring BBBD in human [8]. IAM generated a prolonged increase in the ratio of oxyhemoglobin $(\mathrm{HbO})$ and deoxyhemoglobin $(\mathrm{HbR})$. In this paper, we further study the effects of BBB opening and show cerebral hemoglobin and water concentration changes during BBB opening in mouse and compare these responses between two BBB opening mechanisms.

\section{METHODOLOGY}

\section{1 fNIRS monitoring}

In our fNIRS setup, optical fibre having three light source inputs and one output tip was used as a source. This enables mixing of three narrow band wavelengths, each modulated at a specific frequency [20,21]. Light sources are high-power light emitting diodes having wavelengths of $690 \mathrm{~nm}, 830 \mathrm{~nm}$ and $980 \mathrm{~nm}$. Two fibre detectors were attached in ear canals and the source fibre was placed on middle of the head, see Figure 1. The diameter of the fibre tips are $2.5 \mathrm{~mm}$. Distance between the source and the detectors in sensor placement is roughly $1 \mathrm{~cm}$, enabling measurement of whole brain NIRS effect, rather than only surface of the brain cortex when using a shorter source-detector distance [14]. All fibres have the same physical dimensions.

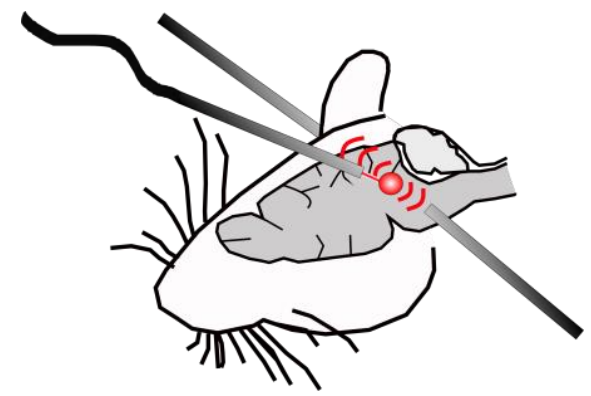

Figure 1. Placement of the fibre sensors. Two fibre detectors are attached in ear canals and the source fibre is places on middle of the head. 
Before starting the analysis, the protocol was translated into a code in Matlab 2019b for plotting purpose. It included setting time for injections of IAM, microbubbles, Evans blue and FUS. A 5th-order Chebyshev LPF at $5 \mathrm{~Hz}$ was applied prior further process. $\mathrm{HbO}, \mathrm{HbR}$, and water concentrations were calculated using the modified Beer-Lamber law (MBLL) [22] and analysis model described by Myllylä et al. [23].

\subsection{BBB opening procedure in mouse}

\section{Mannitol}

Laboratory Mice (CD-1 strain) were anesthetized with ketamine-xylatsine-asepromatsine. External carotid artery was exposed and thin $(<200 \mu \mathrm{m})$ heparine filled polyethylene cannula was introduced into the vessel. $360 \mu \mathrm{l}$ of pre-warmed $25 \%$ mannitol was infused into carotid artery at rate of $720 \mu \mathrm{l} / \mathrm{min} .3 \mathrm{~min}$ post IAM $200 \mu \mathrm{l} 2 \%$ (W/V in $0,9 \%$ saline) Evans blue was infused into tail vein to verify BBB opening after termination of experiment.

\section{FUS}

Mice are anesthetized ketamine-xylazine-acepromazine. Ring-shaped transducer was used, a FUS system RK-20 (Fusinstruments), that operates at 1.1-1.2 MHz frequency. Acoustic pressure at the focused area was up to $1 \mathrm{MPa}$. FUS transducer was modulated at $1.1 \mathrm{MHz}$ frequency having focused length of from 1.5 to $4.5 \mathrm{~mm}$ and focused area of up to $5 \mathrm{~mm}$ (with 70\% acoustic energy). The skin was removed above the skull and the ring transducer mounted on the skull with $1 \%$ agarose.

$10 \mathrm{sec}$ before sonication $200 \mu 1$ of Sonovue microbubbles were injected into tail vein in 1:3 or 1:10 dilution in $0,9 \%$ saline were injected from mouse tail vein 10 seconds before ultrasonic emission. Ultrasonic modulation total exposure time was 1-2 min. As with IAM, Evans blue dye (2\%, w/vol) was used to verify BBB opening.

Animal experiments were performed under the licence of National Animal Experiment Board of Finland (License number: Esavi/2362/04.10.07/2017 and Esavi/826/04.10.07/2018).

\section{RESULTS}

Figures 2 and 3 show typical hemodynamic and water responses for IAM and FUS induced BBB opening in mouse.

\section{IAM BBBD}

\section{Timing}

$60 \mathrm{~s}$ : injection of mannitol

90 s: injection of mannitol ends

(240 s: Injection of Evans blue)
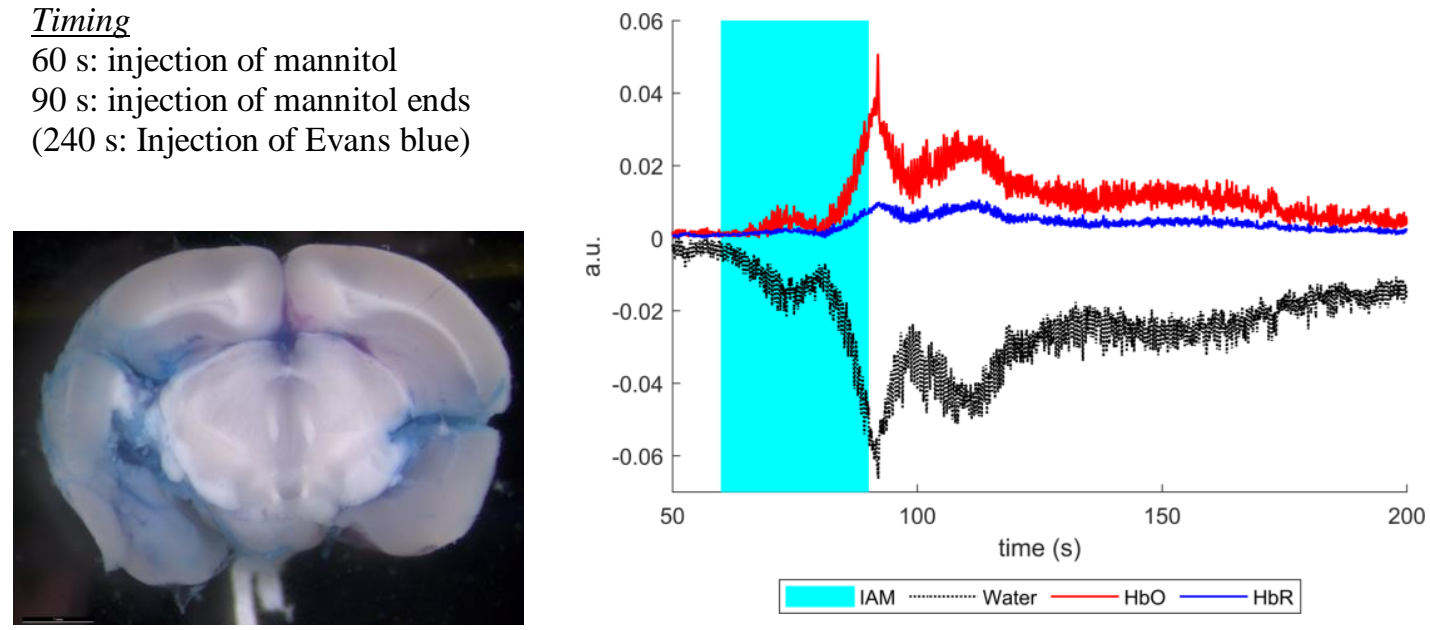

Figure 2. IAM based BBB opening in mouse brain. On the left, Evans blue extravasation associated with BBB opening is visible as blue hue in brain parenchyma. On the right, hemodynamic and water responses for IAM. 
It is supposed that IAM causes osmotic shrinkage of endothelial cells and consequent opening of BBB. This seems to cause remarkable decrease in water concentration from the brain. This is accompanied with vasodilation and hyperemic response explaining increase in $\mathrm{HbO}$ and drop in $\mathrm{HbR}$, showing strong anti-correlation between water and $\mathrm{HbO}$ signals.

\section{FUS BBBD}

\section{$\underline{\text { Timing }}$}

$60 \mathrm{~s}$ : injection of Evans blue

150 s: injection of Sonovue bubbles

$160 \mathrm{~s}$ : FUS is set on

270 s: FUS is set off

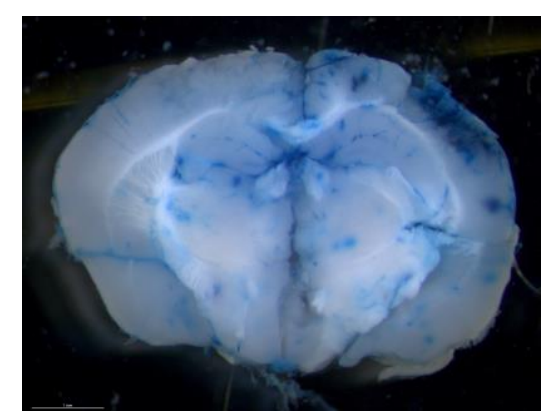

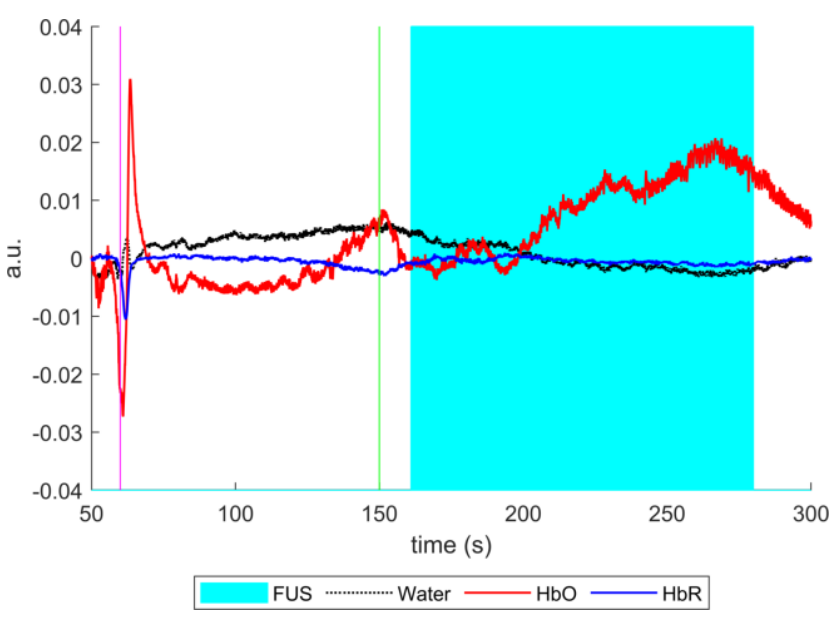

Figure 3. BBBD induced by FUS. On the left, Evans blue extravasation following BBB opening induced by FUS. On the right, hemodynamic and water responses for FUS. Note: high peak visible in fNIRS signal at $60 \mathrm{~s}$ is motion artefact caused by the Evans blue injection.

Earlier studies have shown that FUS can cause neuromodulation depending on the rate of acoustic energy deposition [14]. In Figure 3, shown $\mathrm{HbO} / \mathrm{HbR}$ pattern is similar to the general hemodynamic response to neural activity thus indicating also an effect of neuromodulation. However, it still remains open, and requires further studies to evaluate if there are noticeable differences in $\mathrm{HbO}, \mathrm{HbR}$ and water signals between FUS neuromodulation and FUS BBB opening.

Interestingly, when compared to IAM, in FUS BBB opening we could not detect noticeable change in HbR and water concentration which indicates that BBB opening induced by FUS has different mechanism than IAM based BBB opening.

\section{CONCLUSION}

To our knowledge, this was the first time when hemodynamic and water concentration changes have been measured during BBB opening and compared between FUS and IAM based techniques. Evidently, IAM based BBB opening causes noticeable immediate changes in both haemoglobin and water concentrations. On the contrary, FUS BBBD causes noticeable immediate changes only in $\mathrm{HbO}$ indicating differences between the BBB opening mechanisms. Also the water reaction was different in IAM and FUS methods. In both cases BBB opening was verified by using Evans blue. However, further studies are still required to be performed, in order to visualise and validate more the measured effects of BBB opening.

\section{ACKNOWLEDGEMENTS}

This work was supported by Academy of Finland (grants 314502 and 318347). 


\section{REFERENCES}

[1] Daneman, R. and Prat, A. , "The blood-brain barrier," Cold Spring Harb Perspect.Biol. 7(1), a020412 (2015).

[2] Rasmussen, M. K., Mestre, H. and Nedergaard, M. , "The glymphatic pathway in neurological disorders," The Lancet Neurology 17(11), 1016-1024 (2018).

[3] Lipsman, N., Meng, Y., Bethune, A. J., Huang, Y., Lam, B., Masellis, M., Herrmann, N., Heyn, C., Aubert, I. and Boutet, A. , "Blood-brain barrier opening in Alzheimer's disease using MR-guided focused ultrasound," Nature communications 9(1), 1-8 (2018).

[4] Semyachkina-Glushkovskaya, O., Chehonin, V., Borisova, E., Fedosov, I., Namykin, A., Abdurashitov, A., Shirokov, A., Khlebtsov, B., Lyubun, Y. and Navolokin, N. , "Photodynamic opening of the blood-brain barrier and pathways of brain clearing," Journal of biophotonics 11(8), e201700287 (2018).

[5] Semyachkina-Glushkovskaya, O., Abdurashitov, A., Dubrovsky, A., Bragin, D., Bragina, O., Shushunova, N., Maslyakova, G., Navolokin, N., Bucharskaya, A. and Tuchind, V. , "Application of optical coherence tomography for in vivo monitoring of the meningeal lymphatic vessels during opening of blood-brain barrier: mechanisms of brain clearing," J.Biomed.Opt. 22(12), 121719 (2017).

[6] Doolittle, N. D., Muldoon, L. L., Culp, A. Y. and Neuwelt, E. A., "Delivery of chemotherapeutics across the bloodâ€"brain barrier: challenges and advances," 71, 203-243 (2014).

[7] Hynynen, K., McDannold, N., Vykhodtseva, N. and Jolesz, F. A. , "Noninvasive MR imaging-guided focal opening of the blood-brain barrier in rabbits," Radiology 220(3), 640-646 (2001).

[8] Kiviniemi, V., Korhonen, V., Kortelainen, J., Rytky, S., Keinanen, T., Tuovinen, T., Isokangas, M., Sonkajarvi, E., Siniluoto, T., Nikkinen, J., Alahuhta, S., Tervonen, O., Turpeenniemi-Hujanen, T., Myllyla, T., Kuittinen, O. and Voipio, J. , "Real-time monitoring of human blood-brain barrier disruption," PLoS One 12(3), e0174072 (2017).

[9] Liu, H. L., Fan, C. H., Ting, C. Y. and Yeh, C. K. , "Combining microbubbles and ultrasound for drug delivery to brain tumors: current progress and overview," Theranostics 4(4), 432-444 (2014).

[10] Kuittinen, O., Siniluoto, T., Isokangas, M., Turpeenniemi-Hujanen, T., Peltonen, J., Alahuhta, S. and Sonkajarvi, E. , "Chemotherapy in conjunction with blood brain barrier disruption in the treatment of primary central nervous system lymphoma," Duodecim 129(15), 1563-1570 (2013).

[11] Kuitunen, H., Tokola, S., Siniluoto, T., Isokangas, M., Sonkajärvi, E., Alahuhta, S., Turpeenniemi-Hujanen, T., Jantunen, E., Nousiainen, T. and Vasala, K. , "Promising treatment results with blood brain barrier disruption (BBBD) based immunochemotherapy combined with autologous stem cell transplantation (ASCT) in patients with primary central nervous system lymphoma (PCNSL)," J.Neurooncol. 131(2), 293-300 (2017).

[12] Neuwelt, E. A., Maravilla, K. R., Frenkel, E. P., Rapaport, S. I., Hill, S. A. and Barnett, P. A. , "Osmotic bloodbrain barrier disruption. Computerized tomographic monitoring of chemotherapeutic agent delivery," J.Clin.Invest. 64(2), 684-688 (1979).

[13] Munoz, F., Aurup, C., Konofagou, E. E. and Ferrera, V. P. , "Modulation of brain function and behavior by focused ultrasound," Current behavioral neuroscience reports 5(2), 153-164 (2018).

[14] Yoon, K., Lee, W., Lee, J. E., Xu, L., Croce, P., Foley, L. and Yoo, S. S. , "Effects of sonication parameters on transcranial focused ultrasound brain stimulation in an ovine model," PLoS One 14(10), e0224311 (2019).

[15]Chu, P. C., Liu, H. L., Lai, H. Y., Lin, C. Y., Tsai, H. C. and Pei, Y. C. , "Neuromodulation accompanying focused ultrasound-induced blood-brain barrier opening," Scientific Reports 5(1), 1-12 (2015).

[16] Milej, D., Abdalmalak, A., Desjardins, L., Ahmed, H., Lee, T. Y., Diop, M. and Lawrence, K. S. , "Quantification of blood-brain barrier permeability by dynamic contrast-enhanced NIRS," Scientific Reports 7(1), 1-8 (2017).

[17] Todd, N., Zhang, Y., Livingstone, M., Borsook, D. and McDannold, N. , "The neurovascular response is attenuated by focused ultrasound-mediated disruption of the blood-brain barrier," Neuroimage 201, 116010116010 (2019).

[18]Ergin, A., Wang, M., Zhang, J. Y., Bruce, J. N., Fine, R. L., Bigio, I. J. and Joshi, S. , "The feasibility of realtime in vivo optical detection of blood-brain barrier disruption with indocyanine green," J.Neurooncol. 106(3), 551-560 (2012).

[19] Myllylä, T., Kaakinen, M., Zienkiewicz, A., Jukkola, J., Vihriälä, E., Korhonen, V., Kuittinen, O., Eklund, L. and Kiviniemi, V., "Cardiovascular effects of mannitol infusion: a comparison study performed on mouse and human," Biophotonics: Photonic Solutions for Better Health Care VI, 106854A (2018). 
[20] Myllylä, T., Korhonen, V., Suraźyński, Ł, Zienkiewicz, A., Sorvoja, H. and Myllylä, R. , "Measurement of cerebral blood flow and metabolism using high power light-emitting diodes," Measurement 58, 387-393 (2014).

[21] Sorvoja, H., Myllylä, T., Kirillin, M. Y., Sergeeva, E. A., Myllylä, R. A., Elseoud, A., Nikkinen, J., Tervonen, O. and Kiviniemi, V. , "Non-invasive, MRI-compatible fibreoptic device for functional near-IR reflectometry of human brain," Quantum electronics 40(12), 1067 (2010).

[22]Delpy, D. and Cope, M. , "Quantification in tissue near-infrared spectroscopy," Philosophical Transactions of the Royal Society of London.Series B: Biological Sciences 352(1354), 649-659 (1997).

[23] Myllylä, T., Harju, M., Korhonen, V., Bykov, A., Kiviniemi, V. and Meglinski, I. , "Assessment of the dynamics of human glymphatic system by near-infrared spectroscopy," Journal of biophotonics 11(8), e201700123 (2018). 